\title{
In halt.
}

I. Breslan 1862-1880. (Partie Nr. 1-20.) S. 1-19.

$\mathrm{Nr}$.

1. Gegen Mendelsohn . . 5

2. "Mendelsohn . . 6

3. "MENDelsohn . . 7

4. " v. ScHevk . . . 8

5. ,

6. ",

Mendelsohn . . 8

7. ",

v. SchrVe . . . 9

$\begin{array}{lllll}8 . & \text { MaNnheimer } & \cdot & 10 \\ 9 . & \text { MaNNheimer } & \text {. } & 11\end{array}$

v. ScheVr . . . 9

10. " " MANNHEIMER $:$. 12

Nr.

12. " Vоgт . . . . . 13

13. " VoGT . . . . . 14

14. " Mendelsohn . . 14

15. " Mendelsohn . . 15

16. " Mendelsohn . . 15

17. ," RIEMANN . . . . 15

18. " SchotTLïneR . . 17

19. " SсHоTTLÄNDER . . 18

20. " MrNDELSOHN . . 19

II. Berlin 1880-1882. (Partie Nr. 21-35.) S. 20-37.

Nr.

21. Gegen E. S. . . . . 23

2. " S. WINAWER . . 24

23. " B. LASKER . . . 2.

24. " B. LASKER . . . 25

25. "

26. " X. . . . . . . 27

27. " PRIBULsKY . . . 27

28. " W. Сонк . . . . 28

29. " W. Cohs . . . . 29

30. " W. CoHN . . . . 30

31. Böhle, Droysen, B. Las-
Nr.

kER gegen HARMONIST, .

SCHEVE, TARRASCH . . . 30

32. Gegen Münchiorv . . . 32

33. B. Laskfr, v.Scheve, TroBACH gegen HaRMONIST, v. HEyDEBREcK, Dr. 'TAR- 33 RASCH

34. Gegen M. Naumann . . 34

35. v. BARDELEBEN, B. LASKER, Tarrasch gegen Böhlke, NaumanN, v. Scheve . . 35

III. Halle 1882-1885. (Partie Nr. 36-57.) S. 37-63.

36. Gegen Cand. jur. Kuntze $\begin{array}{r}\text { Seite } \\ 39\end{array}$

37.

38. " B. RICHTER . . . 41

39. " KUntze . . . . 42

49. " B. RichteR . . . 43

41. " B. RICHTER . . . 44

42. " B. RICHTER . . . 44

43. " B. RichteR . . . 45

44. , B. RlснтвR . . . 46

45. " B. RichteB . . . 47

46. B. Richter, Schwarz, HolLÄNDER gegen Sickel, R. Schmidt, TarRasch
Nr.

47. Gegen B. Richter . . 49

(4). 49.

50.

51.

$52 . \quad$,

53.

54. ",

$55 . \quad$,

56. ",

57. ,
Jacques Schwarz. 50 W. HAHN . . . 51 BARTHMANN . . . 52 S. LöwenthaL * - 54 v. Scheve + . . 57 F. RiemanN . . . 58 Riemann . . . . 60 C. Schwarzhaupt . 61 H. Schönfuss . . $6 \mathrm{l}$ B. . . . . 62

IV. Das Hamburger Turnier 1885. (Partie Nr. 58-74.) S. 63-96.

$\mathrm{Nr}$.

58.

59 .

60.

61.

62.
Seite

65

68

71

72
$\mathrm{Nr}$.

63. Gegen

64.

65.

66.

67.
Seite

M. BIER . . . . 74

W. PAULSEN . . 76

J. GunsBera $\quad 78$

J. H. BIRD . . . 81

J. Minckwitz . 82 
Nr.

68. Gegen H. v. Gottschall . 83

6!) J. BRRGEB . . . 83

70. " F. RIEMANN . . . 84

71. " J. MasON . . . 87

Nr. Seite

72. Gegen Mackenzie . . 89

73. " SCHOTTLÄNDER . . 92

74. " Blackburne. . . 93

V. Geroldsgrün 1886. (Partie Nr. 75-77.) S. 96-100.

75. Gegen H. v. Gotrschall 98

76. " Dr. Simonsohn . 99

77. Gegen v. Scheve

VI. Das Frankfurter Turnier 1887. (Partie Nr. 78-95.) S. 100-138. Nr.

78. Gegen Schallopp . . . 102

79. " MaCKENZ1E . . . 103

80. " A LAPIN . . . 106

81. " Joh. BERGER : 107

82. " LOUIS PAULSEN . 109

83. " v. BARDBLEBEN . 111

84. " MLACKBURNE . . 112

85. " Gunsbekg . . . 114

86. " A. FRITZ . . . 116

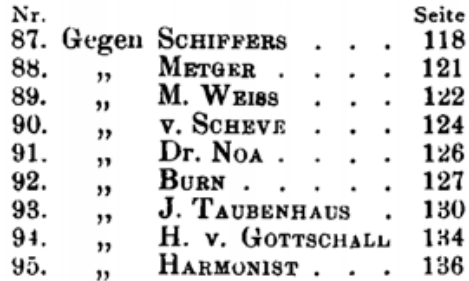

VII. Nürnberg 1887-1888. (Partie Nr. 96-119.) S. 138 161.

Nr.

96 . Gegen G. InIon und M. Seite $\mathrm{Nr}$

$\mathrm{Nr} \quad$ Seite

Kïrschner .

108. Gegen M. Kürschner . 148

97.

W. HAHN . . . 149

98

G. IRION . . . . 139

110.

W. HAHN . . 150

99

Dr. SchWARz , , 140

111.

W. HAHN . . . 15ิ1

100.

M. Kürschner . 140

$11 \%$.

M. KÜrschnek . 152

101 .

M. KÜRSCHNEK

141.113.

К. ЕскаRт . . . 154

102.

M. KÜrschner

142114.

K. Eckart und

103.

M. KüRSChNER - 143

104.

K. Meiser . . . 144

115.

M. Kürschner . $15 b$

105.

K. ECKarT . . . 145

116.

B. Rich'TER . . 156

106.

G. IRION . . . 145

117.

L. Regensburaer . 158

107.

M. KÜRSChNER

146

118 .

M. Rösch . . . 159

M. KÜRSCHNER

$1 4 7 \longdiv { 1 1 9 . }$

M. Rösch : 160

M. KÜRSCHNBR : 161

VIII. Das Nürnberger Turnier 1888. (Partie Nr.120-130.) S.161-205. Nr.

120. Dr. H. v. Gottschall, HARMONIST, Dr. TARRABch gegen J. Metger, J. MieSEs, L. PAULSEN . . . 162

121. Gegen MEtger . . . . 165 Seite $\mathrm{Nr}$.

Nr. Seite

122. " H. v. GotTsCHaLL 167

124. Gegen M. HARMONIST - 178

123.

$1 7 6 \longdiv { 1 3 0 . }$

Louis Paulsen - 180

126. ” J. Metger . . . 186

127. " H. v. GotTschaLL 191

128. ” J. Mieses . . . 194

129. " M. HARMONIST . . 201

IX. Das Leipziger Turnier 1888. (Partie Nr. 131-137.) S. 205-218.

$\mathrm{Nr}$.

13i. Gegen J. Mieses

206 137. v. BARDELEBEN, v. SCHEve,

132. " WILFRIED P PULSEN 209

SCHOTtLÄNDER, Dr. TAR-

133.,$\quad$ v. SCHEVE . . . 210

134. " J. Minckwitz . . 212

135. ” F. RIEMANN . . . 213

136.

v. BARDELEBEN $\quad 214$

RAвсн gegen H. v. GotTschall, Miteies, W. PaulSEN, RIEMANN . . . . 217 
X. Das Breslauer Turnier 1889. (Partie Nr. 138-155.) S. 219-263.

$\mathrm{Nr}$.

138. Gegen Louis Padisen . 223

139. " v. B

140. " J. MetaER . . . 225

141. " HARMONIST . . . 227

142. „ GossIP . . . . 229

143. ” ЈоH. Beroer . . 230

144. $"$ SCHIFFERS . . . 235 15.

145. " J. H. BAUER . . 237 154. " GUNSBerg . . . 259

146. $" M_{\triangle A O N}$. . . . . 238 155.,$\quad$ BURN . . . . . 262

XI. Nürnberg 1889-1890. (Partie Nr. 156-186.) S. 264-291.

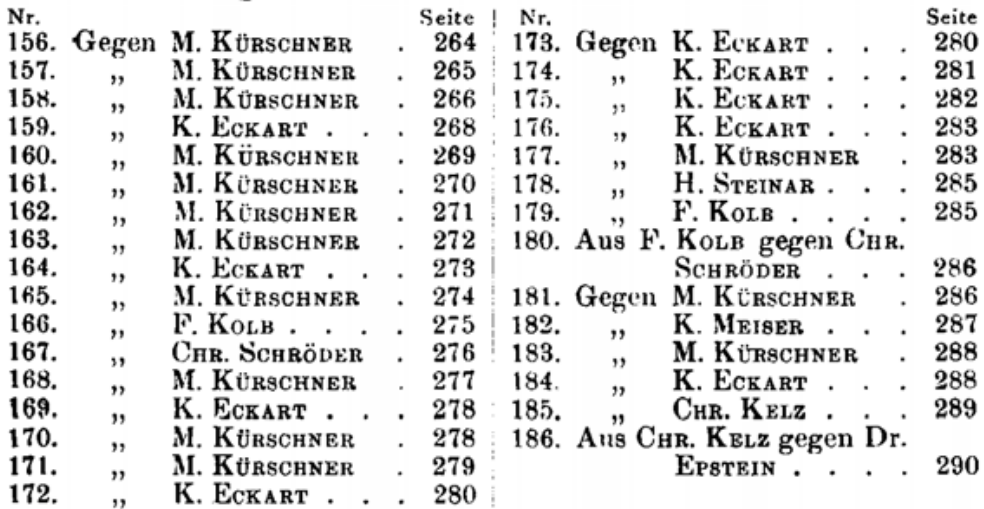

XII. Das Turnier zu Manchester 1890. (PartieNr. 187-203.) S. 291-331.

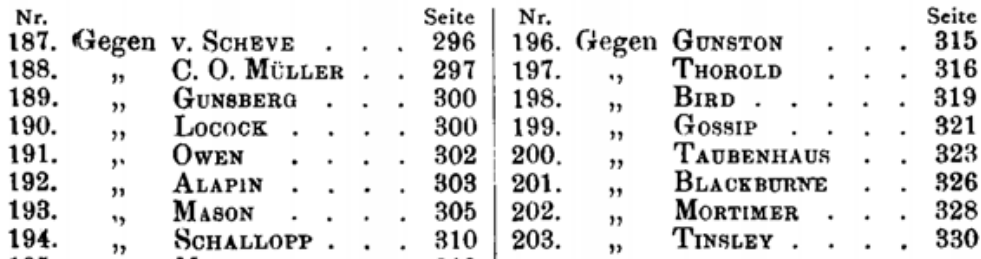

XIII. Nürnberg 1890-1892. (Partie Nr. 204-241.) S. 331-366.

Nr. Seite

204. Gegen A. Strif. . . . 332

205. " A. STEIF . . . . 333

206. " M. KÜRSCHNER. . 335

207. " M. KÜrsCHNRR . 336

208. " M. KÜRSCHNER . 337

209. ” W. HAHN . . . 338

210. ״ W. HABN . . . . 339

211. " W. HAHN . . 340

212. " O. LIEBHARDT . . 342

Nr. Seite

213. Gegen H. Sörgel . . . 343

214. " CHR. KeLZ . . . 343

215. " CHR. KeLZ . . 344

216. " Chr. Kelz . . . 344

217. " Dr. SchWARZ . . 345

218. ” Dr. ScHWARZ . . 345

219. ” CBr. KeLz . . . 345

220. " M. KÜRSCHNBR • 345

221. ” K. ECKART . . . 346 
Seite | Nr. Seite

222. Gegen K. Eckart . . . 348 232. Gegen Taubenhaus . . 355

223. " K. EckarT . . . 349 233. " TAUBRnhaUs . . 355

224. " M. KÜrschner. . 350 234. " Taubenhaus . . 356

225. " K. ECKART . . . 351 235. " TAUBEnHaUS . . 358

226. " W. HAHN . . . 351 236. ”

227. " W. HAHN . . . 351 237. " W. HAHN . . . 360

228. " Chr. Schröder . 351 238. " $\quad$ F. Когв . . . . 362

229. Aus Wirsing geg. Steiner 352 239. " " H. Wirnitzer . . 364

230. Gegen Harmonist . . . 353 240. " Chr. Schröder . 365

231. " HaRmonist . . . 354 i 241. " H. Fiedler . . . 365

XIV. Das Dresdener Turnier 1892. (Partie Nr. 242-255.) S. 366-397.

$\mathrm{Nr}$.

242. Gegen Alapin . . . . 371

243. " Alapin . . . 372

244. " Dr. NOA. . . . 373

245. " J. MakowetZ . . 375

246. " AlbiN . . . . . 378

247. " J. Mieses . . . 380

$\mathrm{Nr}$ Seite

248.

Porges . . . . 382

249. Gegen

383

250. $"$ v. BARDELEBEN . $\mathbf{3 8 4}$

251. ” SCHOTTLÄNDER . . 385

252. " v. SCHEVE . . . 386

253. " WILFRIEDPAULSEN 388

254. " H. v. Got'schaLl 390

255. " WiNAWER . . . 393

XV. Nürnberg 1892-1894. (Partie Nr. 256-278.) S. 397-419.

$\mathrm{Nr}$ Seite $\mid \mathrm{Nr}$. Seite 256. Gegen M. Kürschner . 398 268. Gegen Dr. KARL HoL- Seite

257. ” H. RoMBrRa . . 399

258. " H. HIRSCHLER . . 400

259. " H. ROMвER . . 401

260. " H. HäUSLER . . 401

261. " Dr. KARL HOL-

LÄNDRB. . 402

262. " Dr. KARL HoL-

LÄNDER . . . 403

L̈̈NDER . . $\dot{H} \cdot 408$

269. " Dr. KarL Hol-

L ̈̈NDER . . . 409

$270 . \quad, \quad$. ROMBERG . . 410

271. " H. HIRSCHLER . . 411

272. $"$ H. ROMBERG . . 412

273. ” M. KÜRSCHNER . . 413

264. " CHr. ScHRöDER . 405 276. " CHR. SCHRÖDER . 416

265. ” Chr. Schröder . 406 277. ” F. Kolb . . . . 417

266. ” M. KÜRSCHNER . 407 278. ” H. HIRSCHLER . . 417

267. " $"$ H. LaUbManN . . 407

XVI. Wettkampf mit Tschigorin 1893. (Partie Nr.279-300.) S.419-500.

$\mathrm{Nr}$.

279. 1.PartiedesWettkampfes 420

280. 2. " " "

281. 3. " " " " " $\quad 429$

282. 4. " " " " $\quad 433$

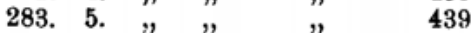

284. 6. " " ,, 441

285. 7. " " " " " $\quad 445$

286. 8. " " " ", 448

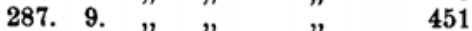

288. $10 ., ", \quad, \quad 454$

289. 11. ", "

456

Nr. Seite

290. 12.PartiedesWettkampfes 461

291. $13 . ", ", 464$

292. 14. " " " " 446

293. 15. " " " $" 471$

294. $16 . \quad 475$

295. 17. " $" 476$

296. 18. " " 480

297. 19. " " 487

298. $20 . " \# \quad " \quad 489$

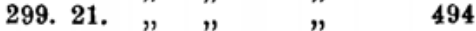

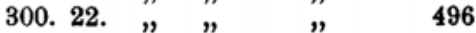

